\title{
COMPUTATION OF THE STABILIZATION PARAMETER FOR THE FINITE ELEMENT SOLUTION OF ADVECTIVE-DIFFUSIVE PROBLEMS
}

\author{
EUGENIO OÑATE, ${ }^{*}{ }^{*}$ JULIO GARCÍA ${ }^{1}$ AND SERGIO IDELSOHN ${ }^{1,2}$ \\ ${ }^{1}$ International Center for Numerical Methods in Engineering (CIMNE), Edificio C1, Gran Capitán s/n, 08034 Barcelona, \\ Spain; \\ ${ }^{2}$ Universidad Nacional del Liboral, Santa Fe, Argentina
}

\begin{abstract}
SUMMARY
In a previous paper a general procedure for deriving stabilized finite element schemes for advective type problems based on invoking higher order balance laws over finite size domains was presented. This provides an expression for the element stabilization parameter in terms of the solution residual and its first derivatives in a kind of iterative or adaptative manner. Details of the application of this procedure to 1D and 2D advectivediffusive problems are given. Some examples of applications showing the potential of the new approach are presented. (C) 1997 John Wiley \& Sons, Ltd.
\end{abstract}

Int. J. Numer. Meth. Fluids, 25: 1385-1407 (1997)

No. of Figures: $10 . \quad$ No. of Tables: $0 . \quad$ No. of References: 30.

KEY WORDS: advective-diffusive problems; stabilization parameter; finite elements

\section{INTRODUCTION}

It is well known that the Galerkin finite element solution of the standard advective-diffusive transport equation becomes unstable for moderate and high values of the advection terms. Traditional ways to eliminate the resulting spurious oscillations are based on the direct addition of some balancing diffusion to the original problem. ${ }^{1-5}$ More rigorous techniques based on Petrov-Galerkin weighting (such as the SUPG and the GLS methods, ${ }^{6,13}$ Taylor-Galerkin ${ }^{14}$ and characteristic approximations ${ }^{15,16}$ have been proposed to derive stabilized finite element equations for advective-diffusive problems. A comparison of these methods can be found in Reference 17. All these introduce (at least) one parameter (usually known as the stabilization parameter, characteristic length, intrinsic time, etc.) whose value controls the stability of the numerical solution.

The precise computation of the hereinafter called stabilization parameter can only be attempted for very simple one-dimensional (1D) problems such as the sourceless advection-diffusion case. Both the 'optimum' stabilization parameter yielding the exact solution at the nodes and the critical one ensuring a stable solution have been found analytically for linear and quadratic elements. ${ }^{2,4,18-20}$ The expressions for the simple 1D case have been heuristically extended for use in two- and three-

Correspondence to: E. Oñate, International Center for Numerical Methods in Engineering (CIMNE), Edificio C1, Gran Capitán s/n, E-08034 Barcelona, Spain. 
dimensional advective-diffusive transport and fluid flow problems in the last two decades. ${ }^{1,2,6-10}$ Indeed, the precise derivation of the stabilization parameters in these cases is nowadays a pending issue.

One of the first attempts to evaluate the stabilization parameter independently of the 1D expression was presented by Idelsohn ${ }^{21}$ using a pseudovariational principle. In a recent paper Hughes ${ }^{22}$ has presented the origins of stabilized methods as a particular class of the so-called subgrid-scale models. As a result of this approach an analytical formula for the stabilization parameter involving the element Green function is proposed. The exact computation of this function, however, is as difficult as the original problem itself and an approximate form based on the use of bubble shape functions is proposed. Good results for the simple 1D case are obtained with this approach as reported in Reference 22. This line of thought has been followed by Brezzi and co-workers, ${ }^{23-26}$ who have extended the ideas of Hughes ${ }^{22}$ by exploiting further the well-known stabilization properties of bubble functions.

In this paper a conceptually different approach for deriving the stabilization parameter is proposed. Oñate ${ }^{27}$ has shown quite recently that the standard forms of the stabilization terms appearing in numerical schemes based on finite element, finite difference, finite volume and finite point methods can be naturally found by introducing a priori the characteristic length in the governing differential equations. The new stabilized form of both the balance equation to be satisfied within the analysis domain and the Neumann boundary conditions can be easily found by using higher-order approximations to model the changes in the advection-diffusion and source terms over a balance

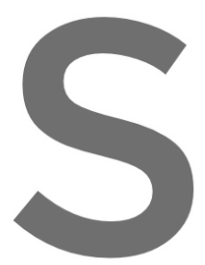
domain. It is interesting differential equations characteristic Galerk In Reference 27 The method is based on derivatives. The express
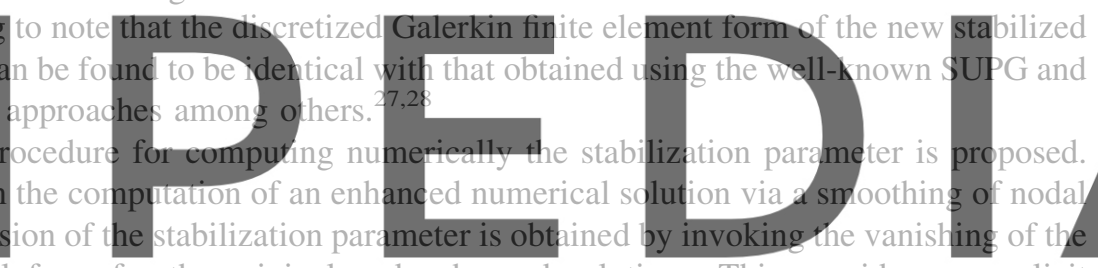
new stabilized residual form for the original and enhanced solutions. This provides an explicit

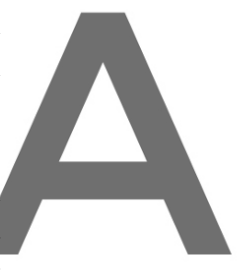
expression for the element stabilization parameter in terms of the standard element residuals and their

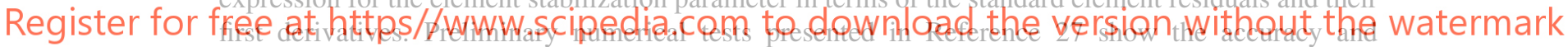
usefulness of the expression found for obtaining stable numerical solutions in an iterative (or $\alpha$ adaptative) manner, where the stabilization parameter $\alpha$ is updated after each solution until a stable solution is found.

The objective of this paper is to explore further the possibilities of the new procedure for computing the stabilization parameter proposed in Reference 27. In the first part of the paper the basis of the method is explained. This includes both the derivation of the stabilized form of the governing differential equation and the expression for the stabilization parameter for one- and two-dimensional advective-diffusive problems. In the second part of the paper the ' $\alpha$-adaptative' procedure for obtaining stable numerical solutions is described and its efficiency and accuracy are shown in a number of examples of application.

\section{STABILIZED FORM OF THE GOVERNING EQUATIONS FOR ADVECTIVE-DIFFUSIVE TRANSPORT}

\subsection{One-dimensional advective-diffusive problems}

Let us consider for simplicity the standard advection-diffusion transport problem to be solved in a one-dimensional domain of length $l$ (Figure 1(a)). Figure 1(b) shows a typical segment AB of length $\overline{\mathrm{AB}}=h$ where balance (equilibrium) of fluxes must be satisfied. The values of the diffusive flow rate 
(a)

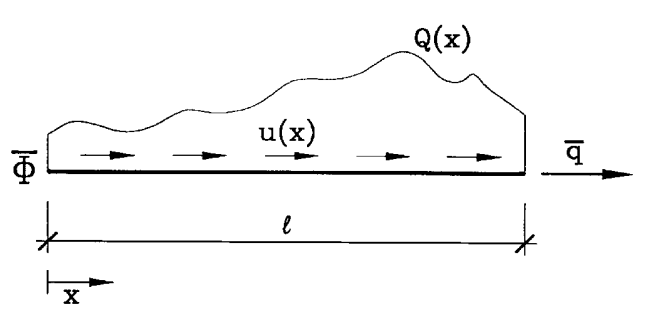

(b)

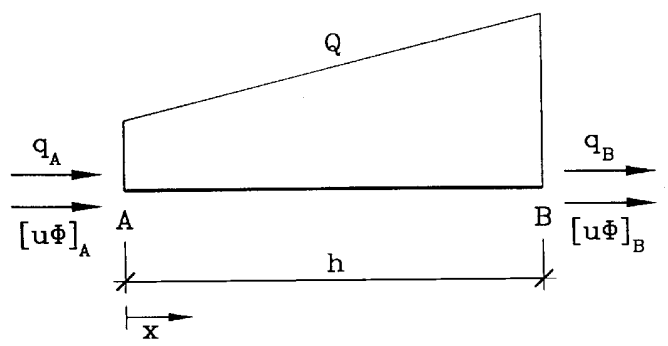

Figure 1. (a) One-dimensional convection-diffusion problem. (b) Finite balance domain AB

$q$ and the advective transport rate $u \phi$ at a point $\mathrm{A}$ with co-ordinate $x_{\mathrm{A}}=x_{\mathrm{B}}-h$ can be approximated in terms of values at point B using the higher-order expansions

$$
\begin{aligned}
{[u \phi]\left(x_{\mathrm{B}}-h\right) } & =[u \phi]\left(x_{\mathrm{B}}\right)-\left.h \frac{\mathrm{d}[u \phi]}{\mathrm{d} x}\right|_{\mathrm{B}}+\left.\frac{h^{2}}{2} \frac{\mathrm{d}^{2}[u \phi]}{\mathrm{d} x^{2}}\right|_{\mathrm{B}}-O\left(h^{3}\right), \\
q(x-h) & =q(x)-h \frac{\mathrm{d} q}{\mathrm{~d} x}+\frac{h^{2}}{2} \frac{\mathrm{d}^{2} q}{\mathrm{~d} x^{2}}+O\left(h^{3}\right),
\end{aligned}
$$
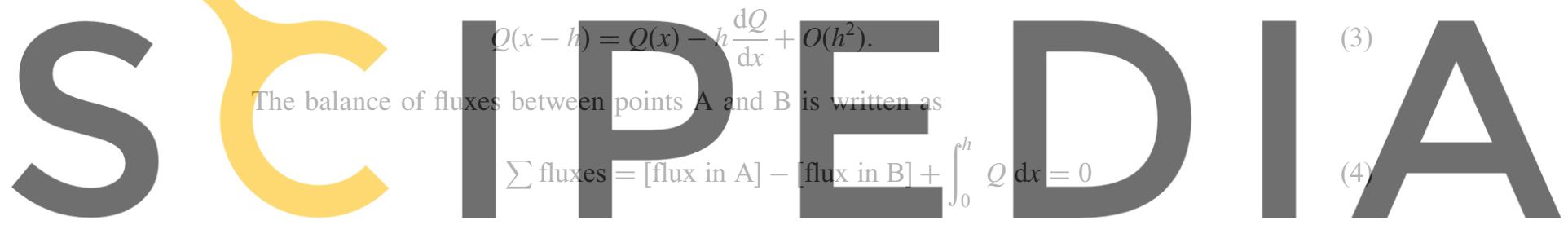

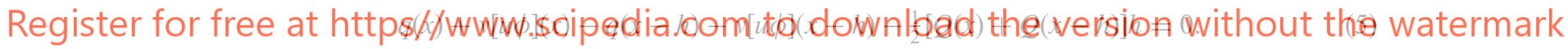

In (1)-(5) $\phi$ is the unknown transported variable, $u$ is the known velocity field which is taken as positive if it acts in the direction of the $x$-axis, $v$ is the advective material parameter which is assumed to be constant and $Q$ is a distributed source which is assumed here to have a linear distribution over the domain $\mathrm{AB}$.

Substituting (1) into (5) and noting that the position of point B is arbitrary gives after simplification

$$
-v \frac{\mathrm{d}[u \phi]}{\mathrm{d} x}-\frac{\mathrm{d} q}{\mathrm{~d} x}+Q=0 .
$$

Making use now of Fourier's law $q=-k \mathrm{~d} \phi / \mathrm{d} x$ gives finally

$$
r-\frac{h}{2} \frac{\mathrm{d} r}{\mathrm{~d} x}=0, \quad 0<x<l,
$$

with

$$
r=-v \frac{\mathrm{d}(u \phi)}{\mathrm{d} x}+\frac{\mathrm{d}}{\mathrm{d} x}\left(k \frac{\mathrm{d} \phi}{\mathrm{d} x}\right)+Q .
$$

Note that for $h \rightarrow 0$ (i.e. when the length of the balance domain is infinitesimal) the standard form of the governing equation for 1D advective-diffusive transport $(r=0)$ is recovered. 
The essential (Dirichlet) boundary condition is the standard one given by

$$
\phi-\bar{\phi}=0 \quad \text { on } x=0,
$$

where $\bar{\phi}$ is the prescribed unknown field at the Dirichlet boundary.

For consistency the stabilized form of the Neumann boundary condition is needed. This can be obtained by invoking again the balance law in a segment $\mathrm{AB}$ next to a boundary point. For convenience the length of this segment is taken as half of the characteristic length $h$ of the interior domain points. ${ }^{27}$ The balance equation is, assuming now the source $Q$ to be constant over $\mathrm{AB}$ (see Figure 2,

$$
\bar{q}-q\left(x_{\mathrm{A}}\right)-v[u \phi]\left(x_{\mathrm{A}}\right)-\frac{h}{2} Q=0,
$$

where $\bar{q}$ is the prescribed total flux at $x=l$ and $x_{\mathrm{A}}=x_{\mathrm{B}}-h / 2$.

Let us express now the advective and diffusive fluxes at point $\mathrm{A}$ in terms of second-order expansions via (1). After a little algebra we obtain

$$
-v u \phi+k \frac{\mathrm{d} \phi}{\mathrm{d} x}+\bar{q}-\frac{h}{2} r=0 \quad \text { on } x=l,
$$

where $r$ is given by (24). Obviously for $h \rightarrow 0$ the standard form of the Neumann boundary condition
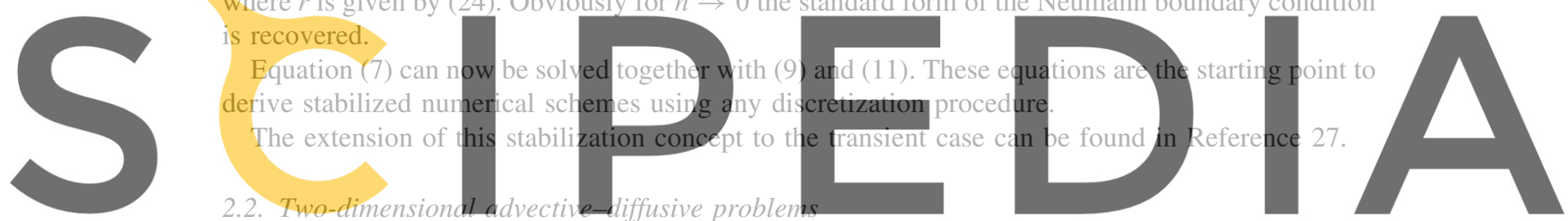

The concepts of the previous subsection will be extended now to the solution of advection-

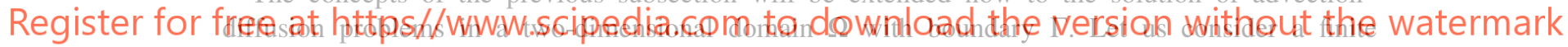
rectangular domain of dimensions $h_{x}$ and $h_{y}$ in directions $x$ and $y$ respectively. Both the advective and diffusive fluxes are assumed to vary linearly along the four sides of the balance domain (Figure 3 ). The flux balance equation will be obtained using the following Taylor expansions: diffusive term, third-order expansion; advective term, third-order expansion; source term, second-order expansion.

The balance of fluxes across the four sides of the rectangular domain of Figure 3 gives after some algebra $^{27}$

$$
r-\frac{1}{2} \mathbf{h}^{\mathrm{T}} \nabla r=0 \quad \text { on } \Omega,
$$

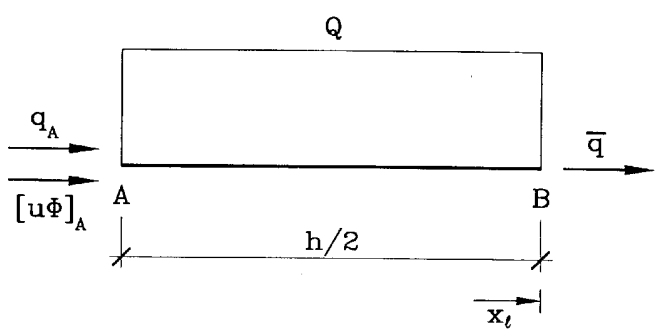

Figure 2. Balance domain next to a Neumann boundary point B 


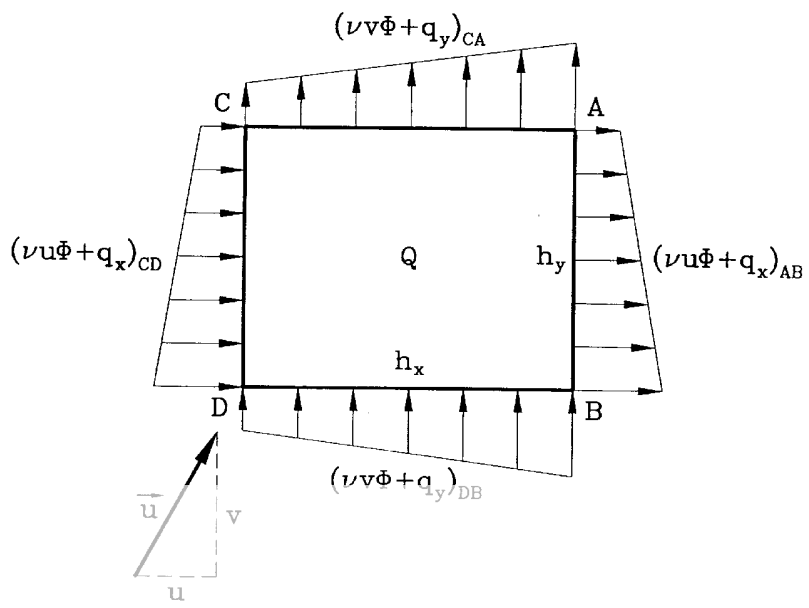

Figure 3. Balance domain for 2D advection-diffusion problem. Advective and diffusive fluxes are assumed to vary linearly along sides
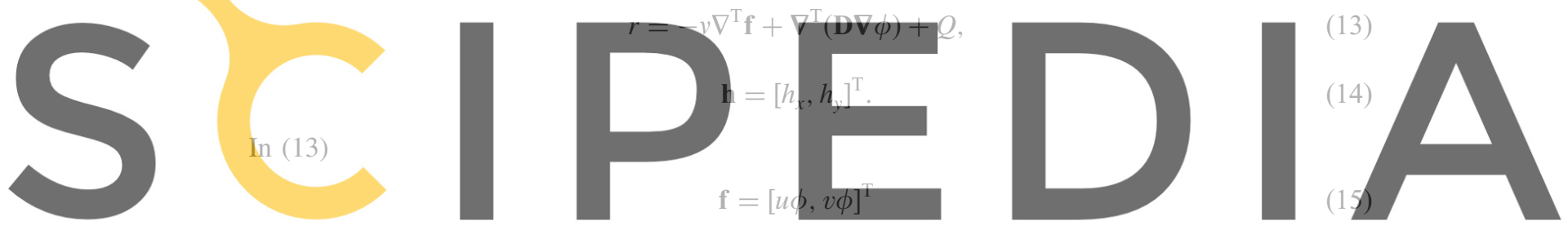

is the advective flux vector,

\section{Register for free at https//www.scipedia.com to ${ }_{2}$ dowwnload the version without the watermark

$$
\nabla=\left[\frac{\partial}{\partial x}, \frac{\partial}{\partial y}\right]
$$

is the gradient operator and

$$
\mathbf{D}=\left[\begin{array}{cc}
k_{x} & 0 \\
0 & k_{y}
\end{array}\right]
$$

is the conductivity matrix. For simplicity $x$ and $y$ are assumed to coincide here with the principal axes of material orthotropy. In (13) $v$ is the advective flux parameter which will be assumed to be constant throughout the domain $\Omega(v=\rho c$ for thermal convection problems). Also as usual the velocities $u$ and $v$ are taken as positive if coincident with the direction of the global axes $x$ and $y$ respectively.

The boundary conditions are written as

$$
\phi-\bar{\phi}=0 \quad \text { on } \Gamma_{\phi},
$$

where $\Gamma_{\phi}$ is the Dirichlet boundary, where the variable is prescribed, and

$$
-v \mathbf{n}^{\mathrm{T}} \mathbf{u} \phi+\mathbf{n}^{\mathrm{T}} \mathbf{D} \boldsymbol{\nabla} \phi+\bar{q}_{n}-\frac{1}{2} \mathbf{h}^{\mathrm{T}} \mathbf{n} r=0 \quad \text { on } \Gamma_{q},
$$

where $\bar{q}_{n}$ is the prescribed total flux across the Neumann boundary $\Gamma_{q}$ with $\Gamma=\Gamma_{\phi} \cup \Gamma_{q}$ and $\mathbf{n}=\left[n_{x}, n_{y}\right]^{\mathrm{T}}$ is the normal vector. 
Equation (19) has been obtained by balance of fluxes in the boundary domain of Figure 4 following the same procedure of the $1 \mathrm{D}$ case previously explained. ${ }^{27,28}$

The standard differential equations are simply obtained by neglecting the stabilizing terms in (12)(19) (i.e. making $h=0$ ), which gives for the steady state case

$$
\begin{aligned}
& r=0 \quad \text { on } \Omega, \quad \phi-\bar{\phi}=0 \quad \text { on } \Gamma_{\phi}, \\
& -v \mathbf{n}^{\mathrm{T}} \mathbf{u} \phi+\mathbf{n}^{\mathrm{T}} \mathbf{D} \boldsymbol{\nabla} \phi+\bar{q}_{n}=0 \quad \text { on } \Gamma_{q} .
\end{aligned}
$$

The extension to three-dimensional problems is straightforward and identical stabilized expressions are obtained.

\subsection{The concept of intrinsic time}

It can be further assumed that $h_{x}=h \cos \alpha$ and $h_{y}=h \sin \alpha$, where $\alpha$ is the angle which the velocity vector $\mathbf{u}=[u, v]^{\mathrm{T}}$ forms with the global $x$-axis and the distance $h=\left(h_{x}^{2}+h_{y}^{2}\right)^{1 / 2}$ is the characteristic length of the 2D advective-diffusive problem. This assumption implies that $\mathbf{h}$ is taken parallel to $\mathbf{u}$. The stabilized governing equation (12) can now be written as

$$
r-\frac{h}{2|\mathbf{u}|} \mathbf{u}^{\mathrm{T}} \nabla r=0
$$

In the derivation of (21) use of the identities $\sin \alpha=u /|\mathbf{u}|$ and $\cos \alpha=v /|\mathbf{u}|$ has been made.

The intrinsic time in 2D advective-diffusive problems is defined
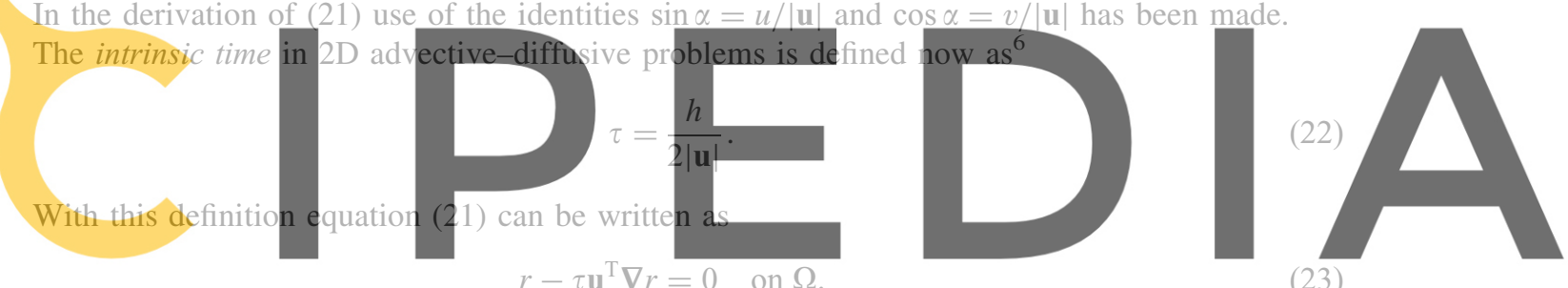

$$
r-\tau \mathbf{u}^{\mathrm{T}} \nabla r=0 \quad \text { on } \Omega
$$

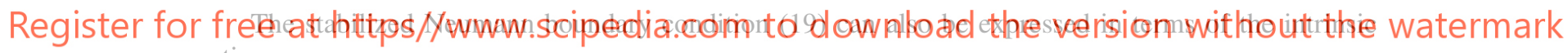
time as

$$
-i \mathbf{n}^{\mathrm{T}} \mathbf{u} \phi+\mathbf{n}^{\mathrm{T}} \mathbf{D} \boldsymbol{\nabla} \phi+\bar{q}_{n}-\tau \mathbf{u}^{\mathrm{T}} \mathbf{n} r=0 \quad \text { on } \Gamma_{q} .
$$

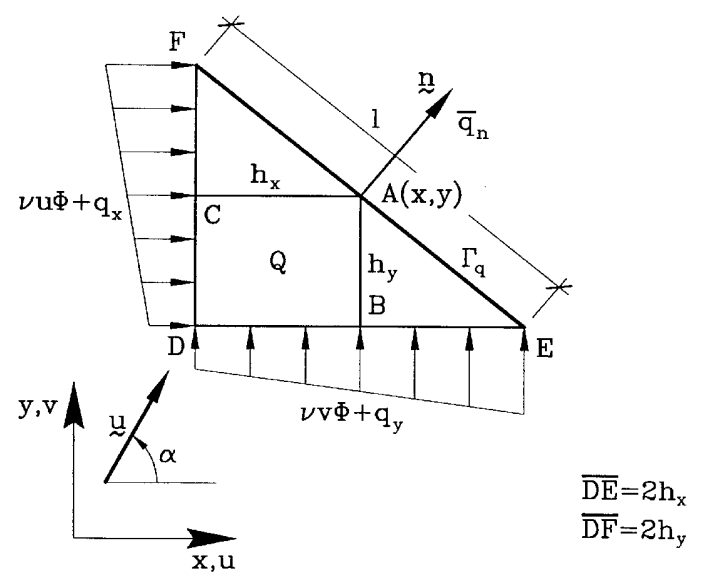

Figure 4. Balance of fluxes in a two-dimensional domain next to a Neumann boundary 


\section{Remark 1}

It is interesting to note that the finite element Galerkin form of the new stabilized differential equations leads to a set of discretized equations identical to those obtained by the standard SUPG approach. $^{27}$ This indicates that the stabilized form of the governing differential equations can be considered as the 'intrinsic' stable expression of the problem, leading to stable numerical schemes for any discretization procedure.

\section{Remark 2}

The assumption of the characteristic length vector $\mathbf{h}$ being parallel to the velocity vector $\mathbf{u}$ is a simplification which eliminates any stabilizing transverse diffusion effect (as is usual in the SUPG approach). Indeed, the two characteristic lengths $h_{\gamma}$ and $h$ can be kent as 'free' stabilization parameters. This can be essential to reproduce the beneficial effect of the so-called transverse (or crosswind) discontinuity-capturing diffusion when sharp layers are present. ${ }^{29}$

\section{A PROCEDURE FOR COMPUTATION OF THE STABILIZATION PARAMETER}

Let us consider the finite element solution of an advective-diffusive problem. The standard interpolation within an element $e$ with $n$ nodes can be written as
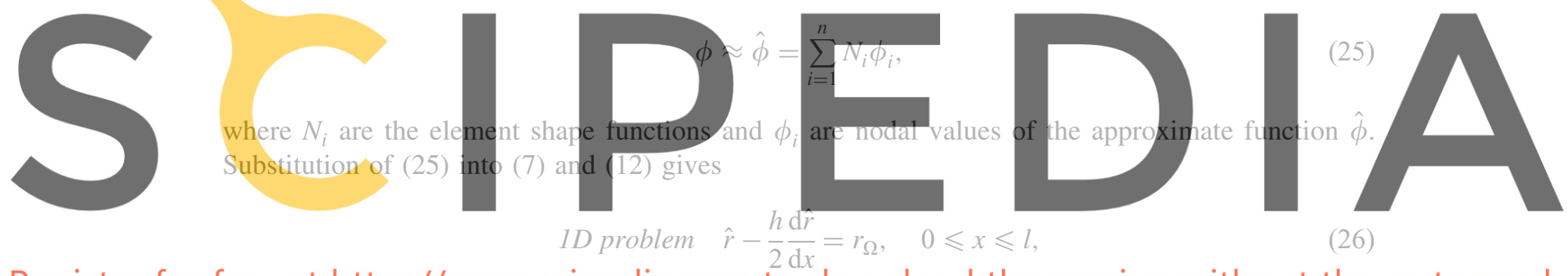

(26)

Register for free at https//www.scipedia.com to download the version without the watermark

$$
2 D \text { problem } \hat{r}-\frac{h}{2|\mathbf{u}|} \mathbf{u}^{\mathrm{T}} \nabla \hat{r}=r_{\Omega} \quad \text { in } \Omega,
$$

where $\hat{r}=r(\hat{\phi})$.

Let us define now the average residual of a particular numerical solution over an element as

$$
r^{(e)}=\frac{1}{\Omega^{(e)}} \int_{\Omega^{(e)}} r_{\Omega} \mathrm{d} \Omega,
$$

where $r_{\Omega}$ is defined by (26) and (27) for the one- and two-dimensional cases respectively. For generality the two-dimensional problem will be considered next.

Substituting (27) into (28) gives

$$
r^{(e)}=\hat{r}^{(e)}-\left(\frac{h}{2|\mathbf{u}|} \mathbf{u}^{\mathrm{T}} \nabla \hat{r}\right)^{(e)},
$$

where

$$
a^{(e)} \equiv \frac{1}{\Omega^{(e)}} \int_{\Omega^{(e)}} a \mathrm{~d} \Omega
$$


For simplicity the value of the characteristic length will be assumed to be constant over the element, i.e. $h=h^{(e)}$. With this assumption eq. (29) can be simplified to

$$
r^{(e)}=\hat{r}^{(e)}-\frac{h^{(e)}}{2}\left(\frac{\mathbf{u}^{\mathrm{T}}}{|\mathbf{u}|} \nabla \hat{r}\right)^{(e)} \text {. }
$$

Obviously (31) can be further simplified if the velocity is taken as constant within an element.

Let us consider now that an enhanced numerical solution has been found for a given finite element mesh. This can be achieved by projecting into the original mesh an improved solution obtained via mesh refinement or, more simply, by global/local smoothing or superconvergent recovery of derivatives. ${ }^{30}$ If $r_{1}^{(e)}$ and $r_{2}^{(e)}$ respectively denote the element residuals of the original and the enhanced numerical solution for a given mesh, then it is obvious that

$$
r_{1}^{(e)}-r_{2}^{(e)} \geqslant 0
$$

Combining (29) and (32) yields an expression for the element characteristic length as

$$
h^{(e)} \geqslant 2\left(\hat{r}_{2}^{(e)}-\hat{r}_{1}^{(e)}\right)\left[\left(\frac{\mathbf{u}^{\mathrm{T}}}{|\mathbf{u}|} \nabla \hat{r}_{2}\right)^{(e)}-\left(\frac{\mathbf{u}^{\mathrm{T}}}{|\mathbf{u}|} \boldsymbol{\nabla} \hat{r}_{1}\right)^{(e)}\right]^{-1} .
$$
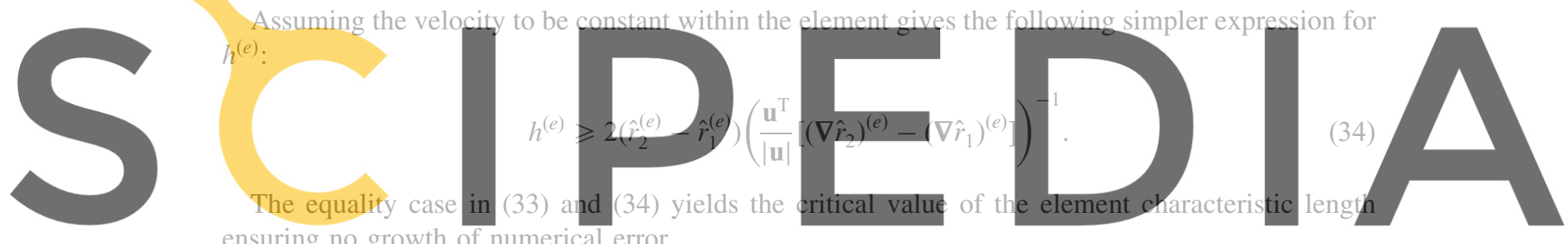

The expression of the intrinsic time parameter for an element can be easily deduced by combining

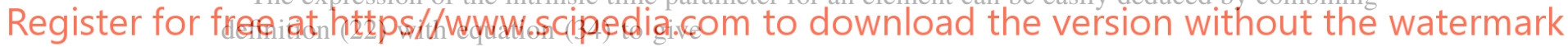

$$
\tau^{(e)} \geqslant\left(\hat{r}_{2}^{(e)}-\hat{r}_{1}^{(e)}\right)\left[\mathbf{u}^{\mathrm{T}}\left(\nabla \hat{r}_{2}^{(e)}-\nabla \hat{r}_{1}^{(e)}\right)\right]^{-1} \cdot
$$

The characteristic length can also be expressed as a proportion of a typical element dimension $l^{(e)}$ as

$$
h^{(e)}=\alpha^{(e)} l^{(e)},
$$

where $\alpha^{(e)}$ is the so-called element 'stabilization parameter'. ${ }^{2}$ The dimension $l^{(e)}$ is usually taken as the element length in 1D problems or the square of the element area in 2D problems, etc. Combining (34) and (36) provides the expression of the element stabilization parameter. These concepts are further clarified with the example presented next.

\section{PROPOSAL FOR AN $\alpha$-ADAPTATIVE STABILIZATION PROCEDURE}

Equations (33)-(36) can be used for deriving an iterative scheme for computation of the element stabilization parameter $\alpha^{(e)}$ as follows.

(i) Solve the stabilized problem defined by (7) and (11) or (12) and (19) by the FEM with an initial guess of the element stabilization parameter $\alpha^{(e)}={ }^{0} \alpha^{(e)}$. 
(ii) Smooth the nodal derivatives field. Evaluate $\hat{r}^{(1)}, \hat{r}^{(2)},(\nabla \hat{r})^{(1)}$ and $(\nabla \hat{r})^{(2)}$ and compute an enhanced value of the stabilization parameter ${ }^{1} \bar{\alpha}^{(e)}$ by (33) and (36).

(iii) Repeat the numerical solution of the stabilized problem with a new value of $\alpha^{(e)}$ given by ${ }^{1} \alpha^{(e)}=\frac{1}{2}\left({ }^{0} \alpha^{(e)}+{ }^{1} \bar{\alpha}^{(e)}\right)$

(iv) Repeat the process until a satisfactory stable numerical solution is found or else $\left\|^{i+1} \alpha^{(e)}-{ }^{i} \alpha^{(e)}\right\| \leqslant \varepsilon$, where $\varepsilon$ is a prescribed tolerance.

In the examples shown next we have found it useful to smooth the distribution of the ${ }^{i} \alpha^{(e)}$-values obtained after step (iii). This can be simply done by a nodal averaging procedure. Also, the number of iterations in the above process is substantially reduced if the initial guess for $\alpha^{(e)}$ is not too far from the final converged value. This can be ensured by using for ${ }^{0} \alpha^{(e)}$ the standard expressions derived from direct extensions of the simple 1D sourceless advective-diffusive case.

Obviously the above scheme applies identically for computing the characteristic length and intrinsic time parameters.

\subsection{Example 1. One-dimensional advective-diffusive problem with no source}

\section{Let us consider the FE solution of the 1D advection-diffusion problem}

$$
-u \frac{\mathrm{d} \phi}{\mathrm{d} x}+k \frac{\mathrm{d}^{2} \phi}{\mathrm{d} x^{2}}=0, \quad 0 \leqslant x \leqslant l
$$
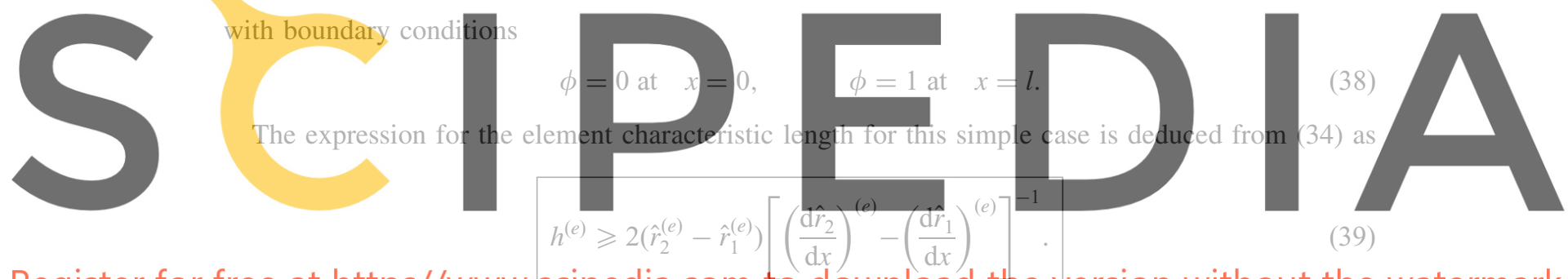

(39)

Register for free at https//www.scipedia.com to download the version without the watermark

The solution will be attempted with the simplest two-node linear element. For a uniform mesh the residual and the residual derivative for an element with nodes $i$ and $i+1$ can be found as

$$
\hat{r}_{1}^{(e)}=-\frac{u}{l^{(e)}}\left(\phi_{i+1}-\phi_{i}\right), \quad\left(\frac{\mathrm{d} \hat{r}_{1}}{\mathrm{~d} x}\right)^{(e)}=0 .
$$

The enhanced solution is obtained now by smoothing the first-order derivative at the nodes. A simple averaging procedure is chosen and the elemental residual for the enhanced solution is given by

$$
\hat{r}_{2}^{(e)}=-\frac{u}{2}\left(\hat{\phi}_{i}^{\prime}+\hat{\phi}_{i+1}^{\prime}\right)+\frac{k}{l^{(e)}}\left(\hat{\phi}_{i+1}^{\prime}-\hat{\phi}_{i}^{\prime}\right),
$$

where $\hat{\phi}_{i}^{\prime}=(\mathrm{d} \hat{\phi} / \mathrm{d} x)_{i}$. Simple algebra gives

$$
\left.\hat{r}_{2}^{(e)}=-\frac{u}{4 l^{(e)}} \phi_{i+1}-\phi_{i-1}+\phi_{i+2}-\phi_{i}\right)+\frac{k}{2\left(l^{(e)}\right)^{2}}\left(\phi_{i+2}-\phi_{i}-\phi_{i+1}+\phi_{i-1}\right) .
$$

A similar procedure leads to

$$
\left(\frac{\mathrm{d} \hat{r}_{2}}{\mathrm{~d} x}\right)^{(e)}=-u \frac{\mathrm{d}^{2} \hat{\phi}}{\mathrm{d} x^{2}}=-\frac{u}{2\left(l^{(e)}\right)^{2}}\left(\phi_{i+2}-\phi_{i}-\phi_{i+1}+\phi_{i-1}\right) .
$$


Substituting (40)-(43) into (39) and using expression (36) gives the element stabilization parameter as

$$
\alpha^{(e)}=\frac{\phi_{i+2}-3 \phi_{i+1}+3 \phi_{i}-\phi_{i-1}}{\phi_{i+2}-\phi_{i+1}-\phi_{i}+\phi_{i-1}}-\frac{1}{\gamma} .
$$

It can be checked that the value of $\alpha^{(e)}$ given by (44) coincides in this case with the analytical expression typically used in practice. For this purpose let us substitute into (44) the general numerical solution for this case given by

$$
\phi_{i}=A+B\left(\frac{1+\gamma(\alpha+1)}{1+\gamma(\alpha-1)}\right)^{i}
$$

where $A$ and $B$ are appropriate constants. After some simple algebra we obtain

$$
\alpha^{(e)}=1-\frac{1}{\gamma}
$$

which coincides with the standard critical value ensuring a stable solution. ${ }^{2,3}$

4.2. Application of the a-adaptive scheme

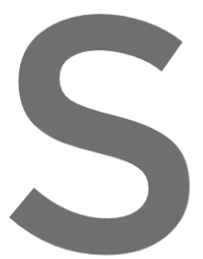

The $\alpha$-adaptative diffusive problem defined by Equation (44) is used iterative process. Note RHS of (44) can be difficult solution are small. This probl
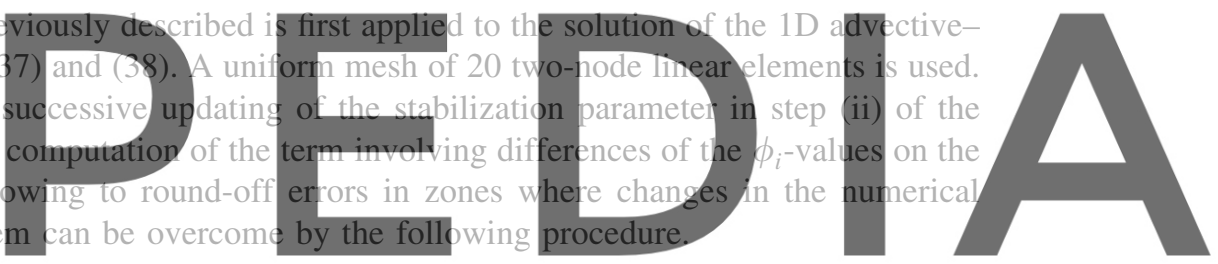

(i) If $\phi_{i+2}-\phi_{0} \leqslant \varepsilon$, where $\phi_{0}$ is the prescribed value at the Dirichlet boundary and $\varepsilon$ is a very

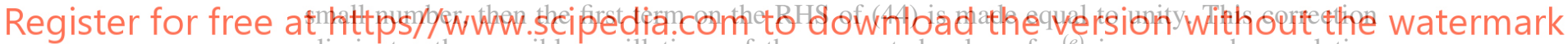
eliminates the possible oscillations of the computed value of $\alpha^{(e)}$ in zones where relative changes in the solution are small. In the examples shown next $\varepsilon=10^{-10}$ has been taken.

(ii) If ${ }^{i} \alpha^{(e)}>1$ or ${ }^{i} \alpha^{(e)}<0$, then ${ }^{i} \alpha^{(e)}=1$ and ${ }^{i} \alpha^{(e)}=0$ are respectively taken.

The first solution is attempted for the Peclet number $\gamma=5$. The initial value ${ }^{0} \alpha^{(e)}=0.5$ is chosen for all elements.

Figure 5 shows the convergence of the solution for $\alpha^{(e)}$. Note that the critical value $\alpha^{(e)}=0.8$ is obtained in all elements after a few iterations. Figure 5 also displays the convergence of the numerical solution for $\phi$, showing convergence to the 'exact' solution after three iterations.

Results for the same problem for $\gamma=25$ are shown in Figure 6. Note that an excellent solution is obtained with just two iterations in this case. Seven iterations are needed, however, to obtain the critical value of $\alpha^{(e)}$ for all elements. Indeed, in both cases it suffices to obtain a good approximation for $\alpha^{(e)}$ in the vicinity of the exit node and this always occurs after two or three iterations.

Figure 7 shows the solution of the same problem for $\gamma=10^{10}$, now with the initial value ${ }^{0} \alpha^{(e)}=0$ for all elements. Excellent results are again obtained after two iterations.

Note that in all three cases analysed the stabilised solution yields the 'quasi-exact' nodal solution giving a localized change in the unknown value from one to (almost) zero over the last element. This can be explained by the well-known fact that the critical stabilization parameter ensuring numerical stability and the optimal one yielding exact nodal results are almost coincident for element Peclet numbers greater than two. 

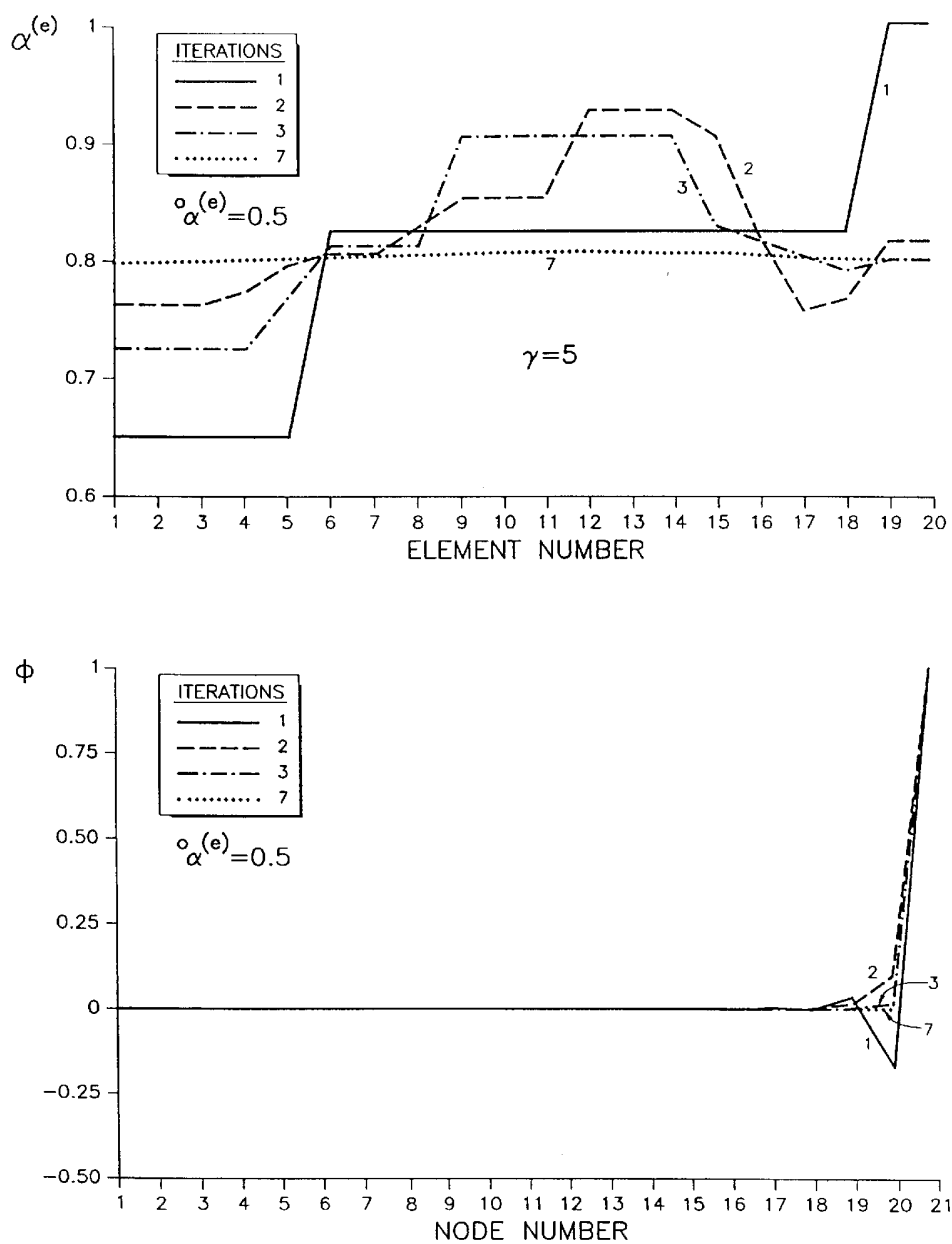

Figure 5. One-dimensional advection-diffusion problem. $Q=0, \phi_{0}=0, \phi_{l}=1$. Convergence of critical value of element stabilization parameter $\alpha^{(e)}$ and of numerical solution $\phi$. Solution obtained with 20 two-node linear elements. Peclet number $\gamma=5,{ }^{0} \alpha^{(e)}=0.5$

\subsection{Example 2. One-dimensional advective-diffusive problem with sine source}

In this example we solve the problem $-u \mathrm{~d} \phi / \mathrm{d} x+k \mathrm{~d}^{2} \phi / \mathrm{d} x^{2}+Q=0$ for $u=1$, $k=0 \cdot 01, Q(x)=\sin (\pi x), l=1$ and $\phi_{0}=\phi_{1}=0$. The interval $[0,1]$ is discretized using 20 twonode linear elements of equal length. This gives an element Peclet number of 2.5. The analytical solution to this problem can be found in Reference 19. The initial value $\alpha^{(e)}=0.0$ is chosen for all elements.

Figure 8(a) shows the evolution of the $\phi$-distribution for the different iterations. Note the expected spurious oscillations obtained for the first solution with wrong values of the stabilization parameter. These oscillations disappear after a few iterations and the final converged solution compares well with the analytical one as shown in Figure 8(b). The relative error between the analytical and the final numerical solution obtained after 10 iterations is plotted in Figure 8(c). Note that the maximum relative error in 10 iterations is only $2 \%$ in the central part of the domain. 

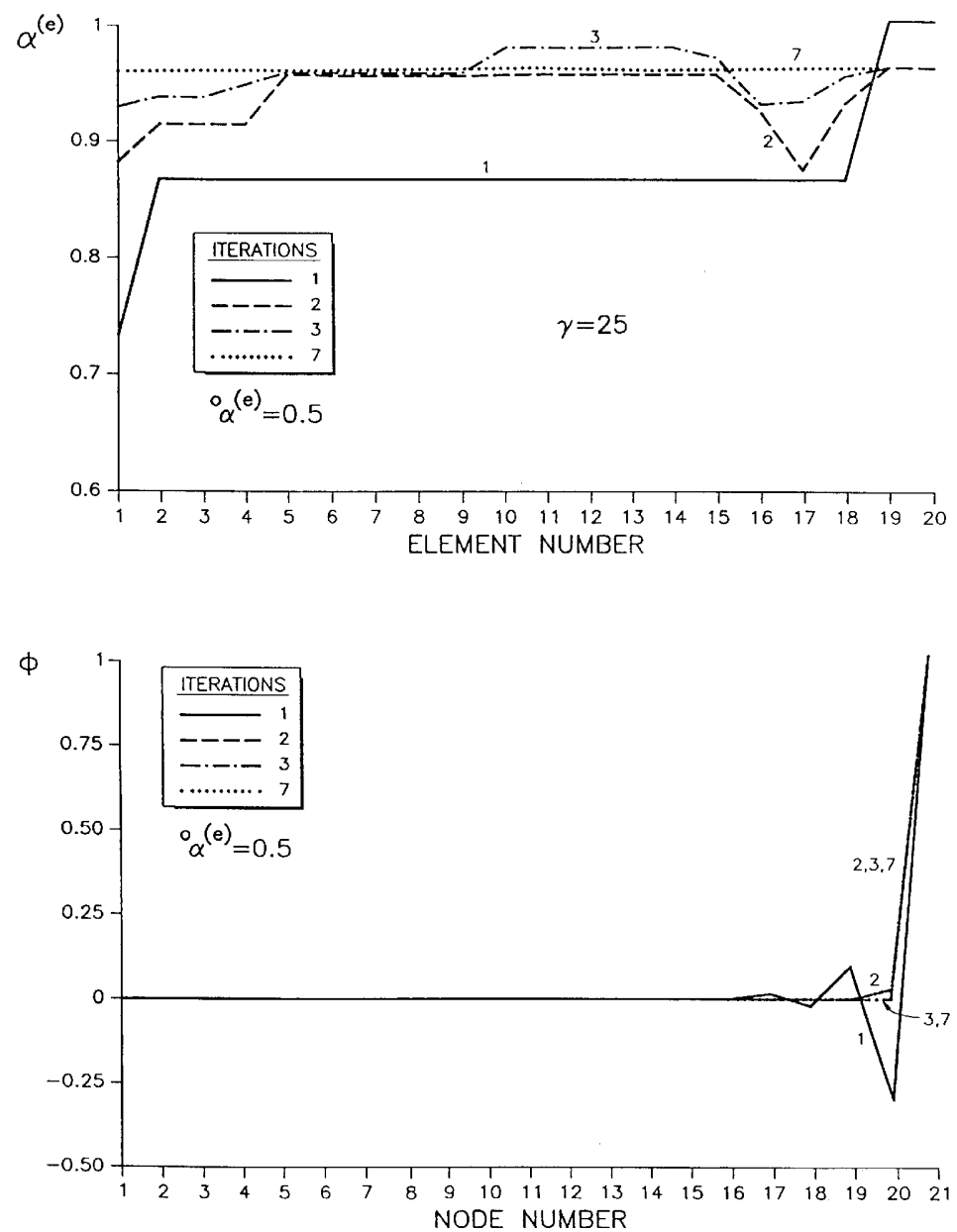

Figure 6. One-dimensional advection-diffusion problem. $Q=0, \phi_{0}=0, \phi_{l}=1$. Convergence of critical value of element stabilization parameter $\alpha^{(e)}$ and of numerical solution $\phi$. Solution obtained with 20 two-node linear elements. Peclet number $\gamma=25,{ }^{0} \alpha^{(e)}=0.5$

The distribution of the element stabilization parameter $\alpha^{(e)}$ for each iteration is shown in Figure $8(\mathrm{~d})$. It is interesting to note that a kind of sinusoidal distribution of $\alpha^{(e)}$ is obtained.

Indeed, the number of iterations can be drastically reduced in this case by choosing a more accurate prediction for the initial stabilization parameter ( $\operatorname{such}$ as ${ }^{0} \alpha^{(e)}=0.5$ ).

\subsection{Example 3. Two-dimensional advective-diffusive problem with constant source and uniform} Dirichlet boundary conditions

The first 2D example chosen is the solution of the standard advection-diffusion equations (20) in a square domain of unit size with

$$
k_{x}=k_{y}=2 \times 10^{-2}, \quad \mathbf{u}=\frac{\sqrt{ } 2}{2}[1,1]^{\mathrm{T}}, \quad Q(x, y)=5 .
$$



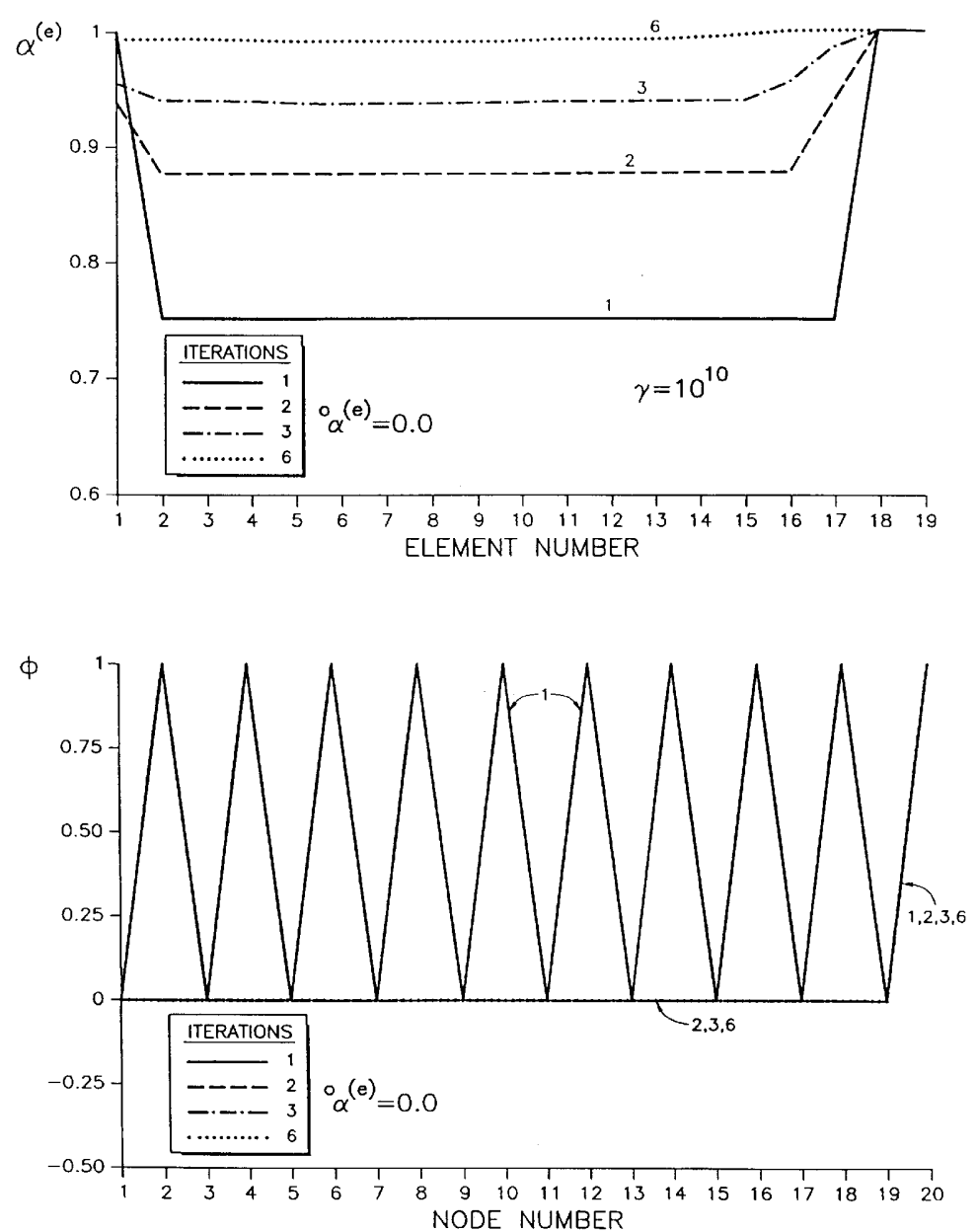

Figure 7. One-dimensional advection-diffusion problem. $Q=0, \phi_{0}=0, \phi_{1}=1$. Convergence of critical value of element stabilization parameter $\alpha^{(e)}$ and of numerical solution $\phi$. Solution obtained with 20 two-node linear elements. Peclet number $\gamma=10^{10},{ }^{0} \alpha^{(e)}=0 \cdot 0$

A Dirichlet boundary condition of $\phi=0$ along the whole domain boundary has been assumed.

The domain has been discretized with a uniform finite element mesh of $20 \times 20$ linear triangles (Figure 9(a)).

The element stabilization parameter $\alpha^{(e)}$ is computed by means of (34) and (36) with $l^{(e)}$ defined as the maximum side length for each triangle. The initial value $\alpha^{(e)}=0.0$ has again been chosen for all elements.

Figure 9(b) shows the distribution of $\phi$ for the first, fifth and final converged solutions (after 10 iterations). Note that a stable solution is found and that the strong initial oscillations are substantially reduced. The evolution of the distribution of the element stabilization parameter is displayed in Figure 9(c). A plot of the final distribution of $\phi$ along the diagonal is shown in Figure 9(d), which is in agreement with Reference 4. 


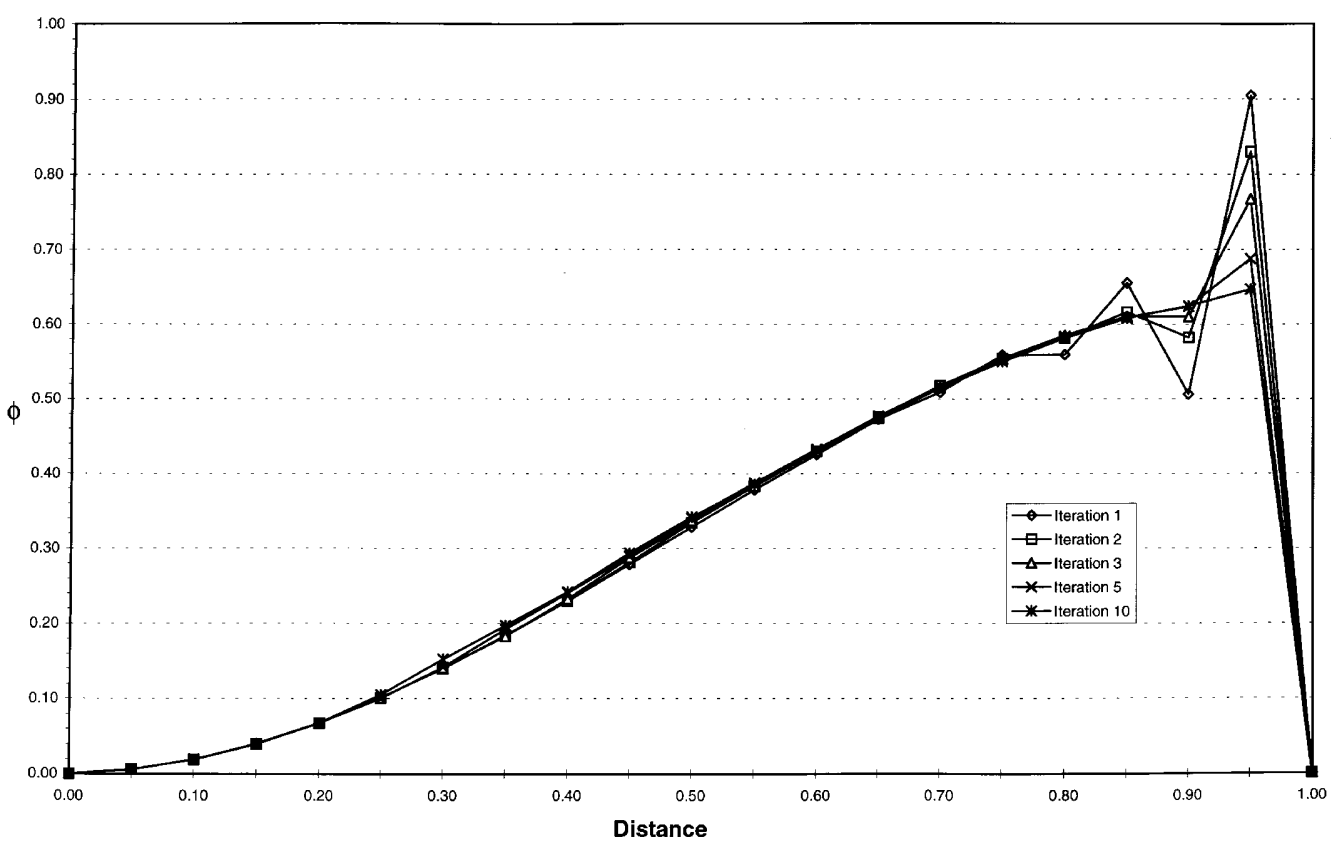

Figure 8(a). Example 2. One-dimensional advection-diffusion problem. $Q=\sin (\pi x), \phi_{0}=\phi_{1}=0$. Convergence of numerical solution obtained with 20 linear elements. Element Peclet number $2 \cdot 5,{ }^{0} \alpha^{(e)}=0 \cdot 0$

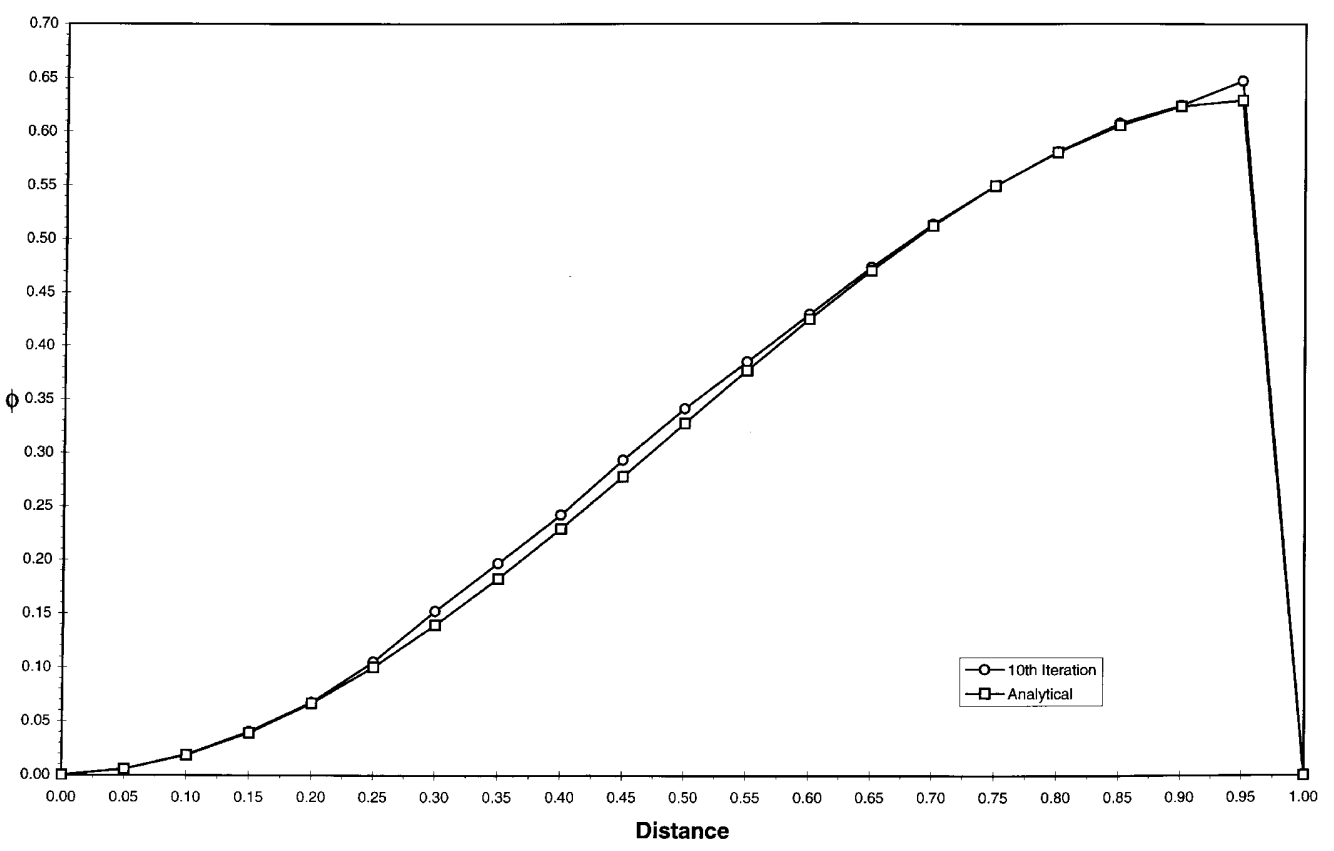

Figure 8(b). Example 2. Comparison of analytical solution and numerical solution (after 10 iterations) 


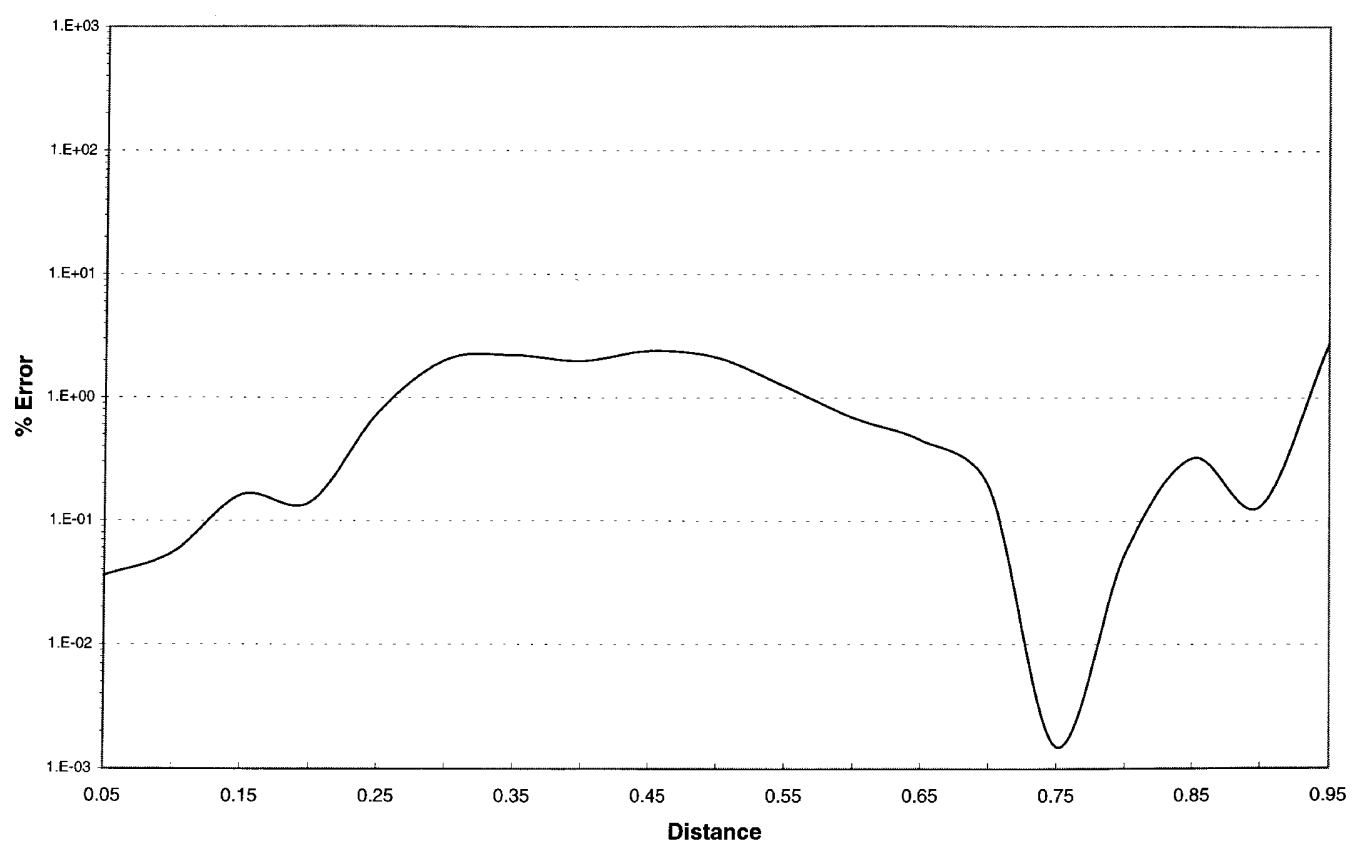

Figure 8(c). Example 2. Percentage error between analytical solution and numerical solution (after 10 iterations)

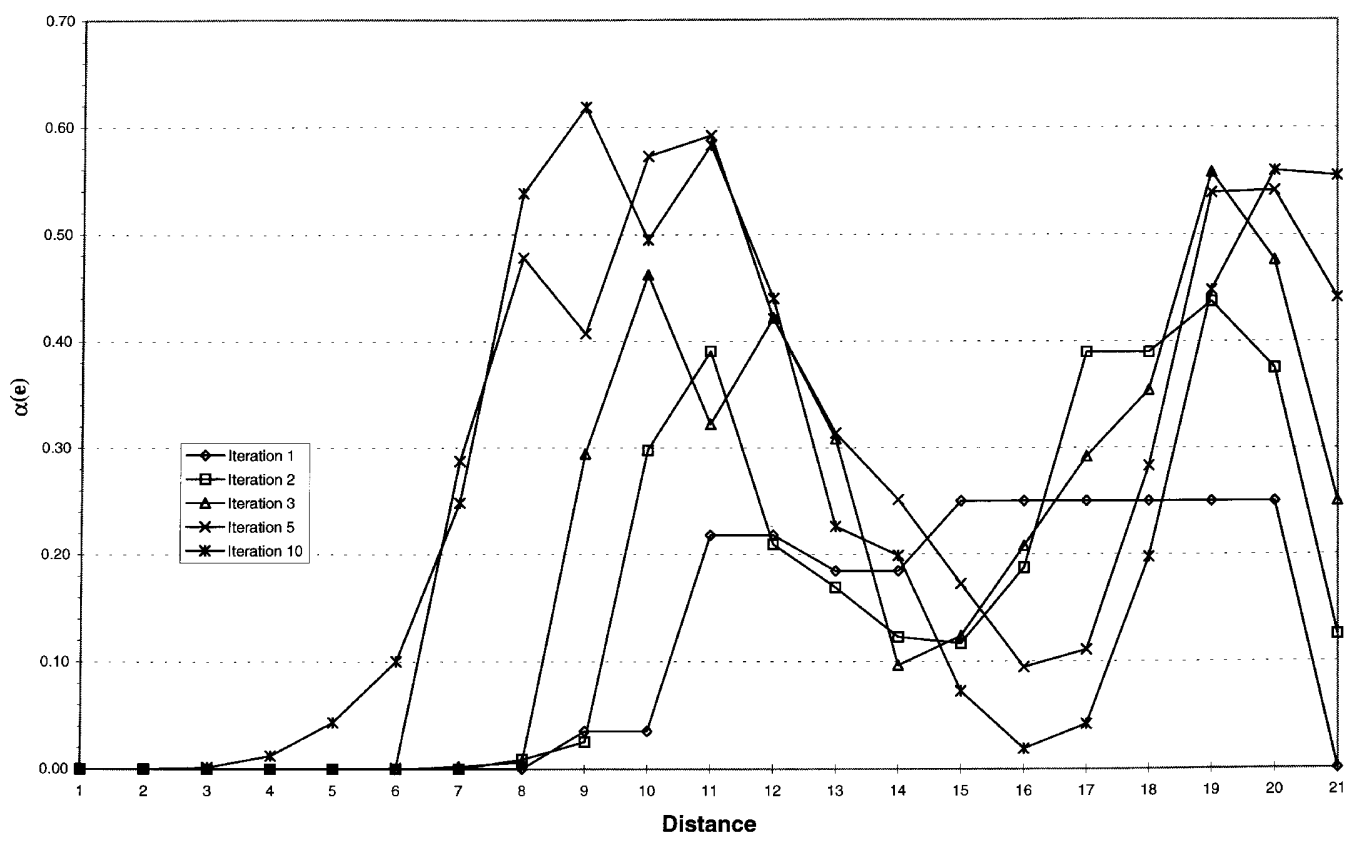

Figure 8(d). Example 2. Distribution of element stabilization parameter $\alpha^{(e)}$ for different iterations 


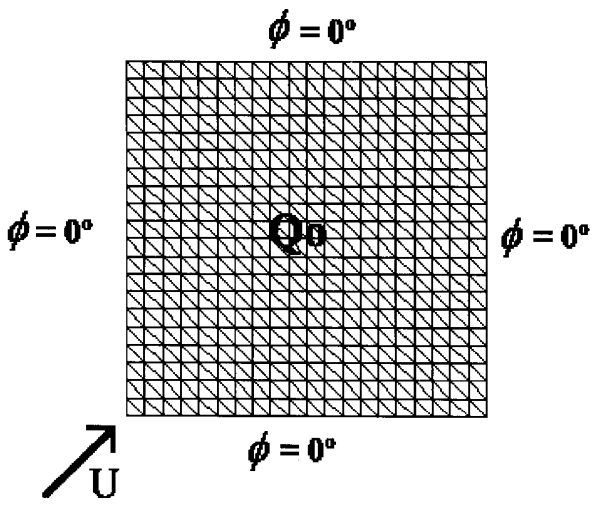

Figure 9(a). Example 3. Two-dimensional advective-diffusive problem with constant source and uniform Dirichlet boundary conditions. Geometry and structured finite element mesh of 800 linear triangles

\subsection{Example 4. Two-dimensional advective-diffusive problem with zero source and non-uniform} Dirichlet boundary conditions

The $2 \mathrm{D}$ advection-diffusion equations are now solved with

$$
\begin{gathered}
\Omega=]-\frac{1}{2}, \frac{1}{2}[\times]-\frac{1}{2},-\frac{1}{2}[, \\
\mathbf{u}=[\cos \theta,-\sin \theta]^{\mathrm{T}}, \\
k_{x}=k_{y}=10^{-6}, \quad Q(x, y)=0, \\
\bar{\phi}(x, y)= \begin{cases}1 & \text { if }(x, y) \in \Gamma_{\phi_{1}}, \\
0 & \text { if }(x, y) \in \Gamma_{\phi_{2}},\end{cases}
\end{gathered}
$$

with $\left.\Gamma_{\phi_{1}}=\left\{-\frac{1}{2}\right\} \times\left[\frac{1}{4}, \frac{1}{2}\right] \cup\right]-\frac{1}{2}, \frac{1}{2}\left[\left\{\times \frac{1}{2}\right\}, \Gamma_{\phi_{2}}=\Gamma_{\phi}-\Gamma_{\phi_{1}}\right.$ and $\Gamma_{q}=0$.

A unstructured finite element mesh of 812 linear triangles has been chosen. The problem has been solved for an angle of the velocity vector given by $\tan \phi=2$ (Figure 10(a)).

The element stabilization parameter has been computed following the procedure of the previous example. Once again the initial value ${ }^{0} \alpha^{(e)}=0$ has been taken.

Figure 10(b) shows the distribution of $\phi$ for the first, fifth and 10th iterations, for which a converged solution is found. Note that the final solution has some overshoots and undershoots as expected. These are due to the inability of the SUPG formulation to deal with sharp layers not perpendicular to the flow direction such as those found in this case. ${ }^{19}$ These oscillations can be eliminated by adding some crosswing dissipation as described in Reference 29. It is interesting to note that the beneficial effect of transverse dissipation in these cases can be modelled by abandoning the assumption that the characteristic vector $\mathbf{h}$ has the same direction as the velocity vector $\mathbf{u}$, as pointed out in Remark 2. Our recent experiences in this context show promising results which will be reported shortly.

The evolution of the distribution of the element stabilization parameter is displayed in Figure $10(c)$. 


\section{Distribution of $\phi$, first iteration}

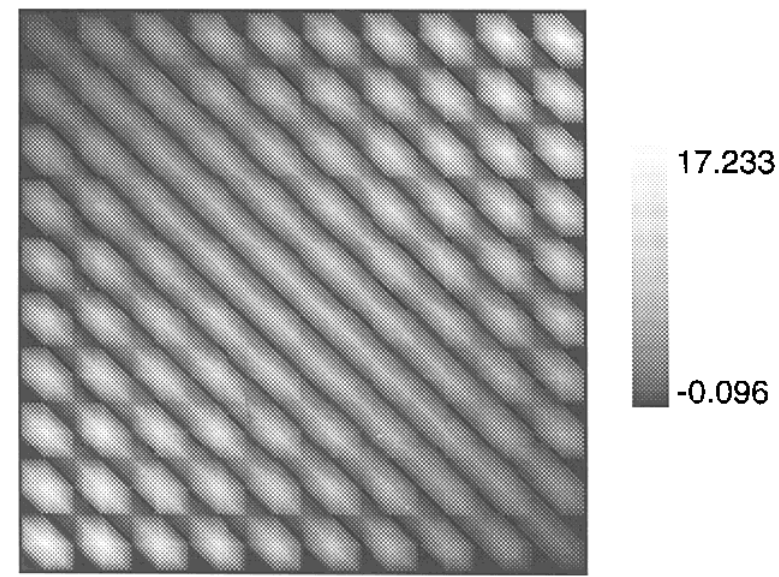

Distribution of $\phi$, fifth iteration

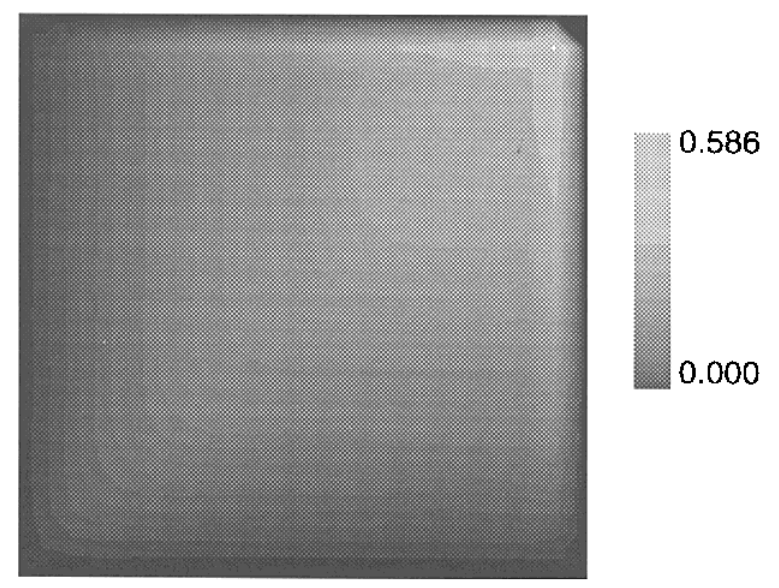

Distribution of $\phi$, tenth iteration

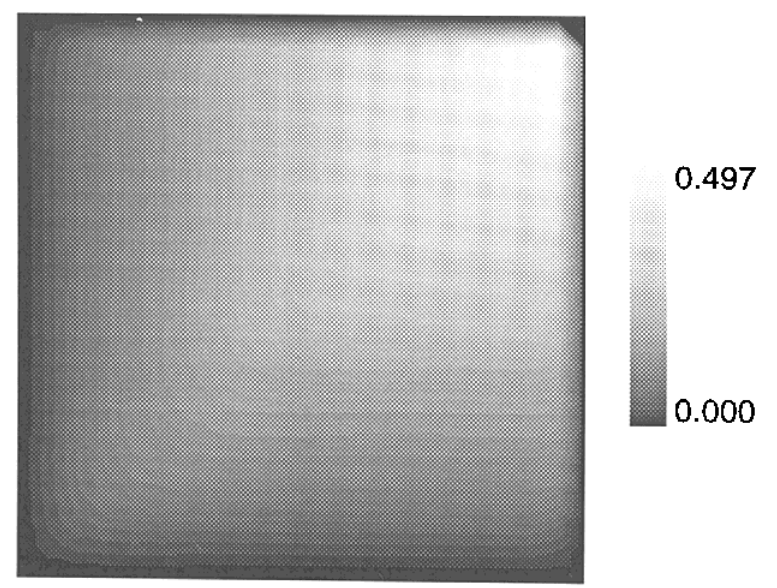

Figure 9(b). Example 3. Evolution of distribution of $\phi$ for different iterations 
Distribution of $\alpha$, first iteration

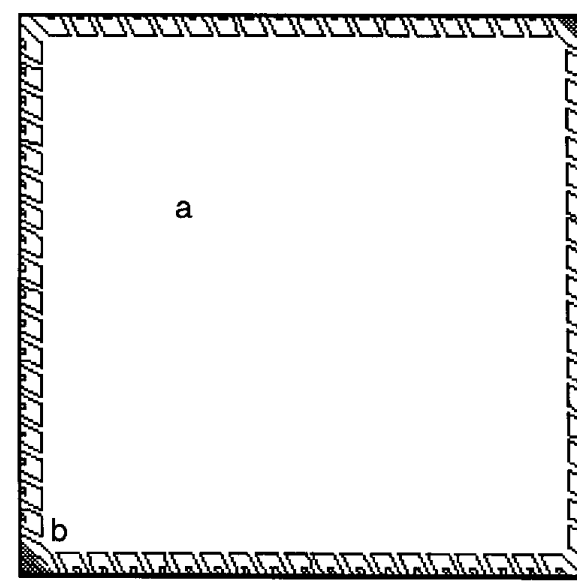

a 0.500

b 0.000

Distribution of $\alpha$, fifth iteration

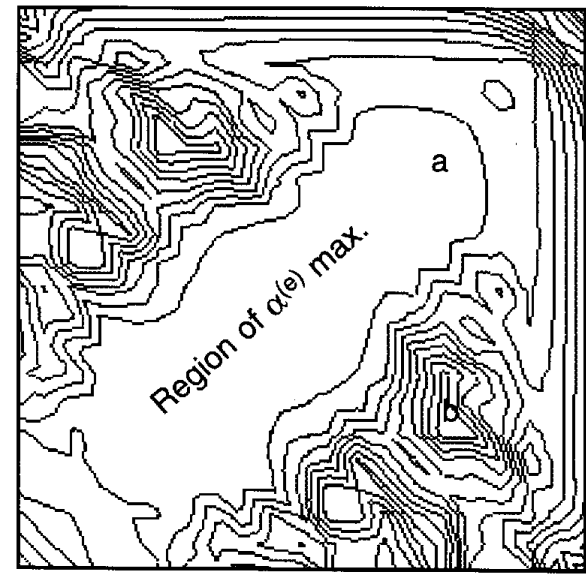

a 0.976

b 0.681

Distribution of $\alpha$, tenth iteration

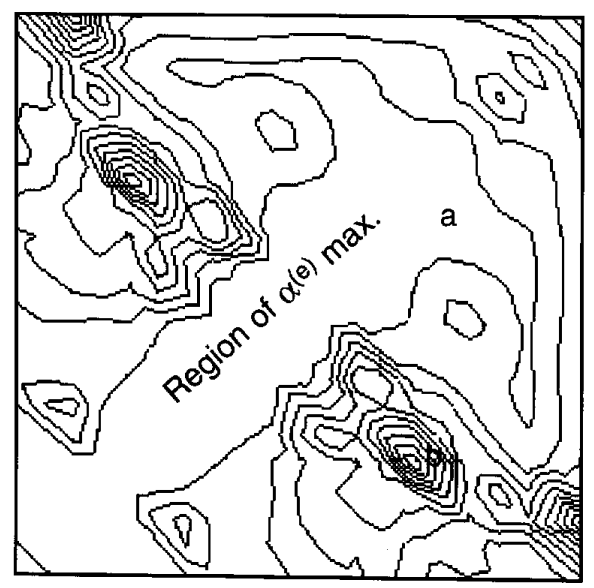

a 0.993

b 0.676

Figure 9(c). Example 3. Evolution of distribution of element stabilization parameter for different iterations 


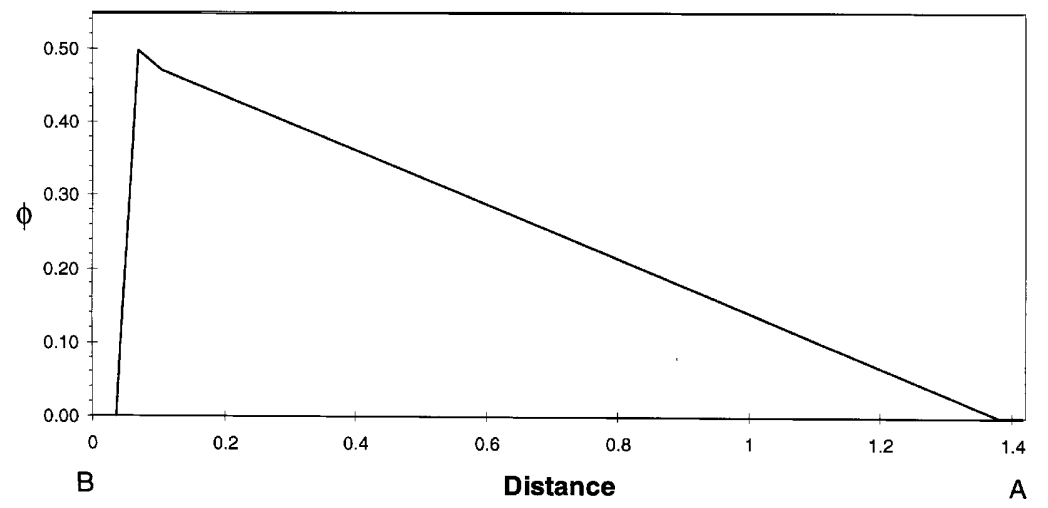

B

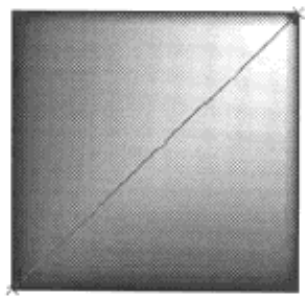

A

Figure 9(d). Example 3. Distribution of $\phi$ along diagonal A-B for last iteration

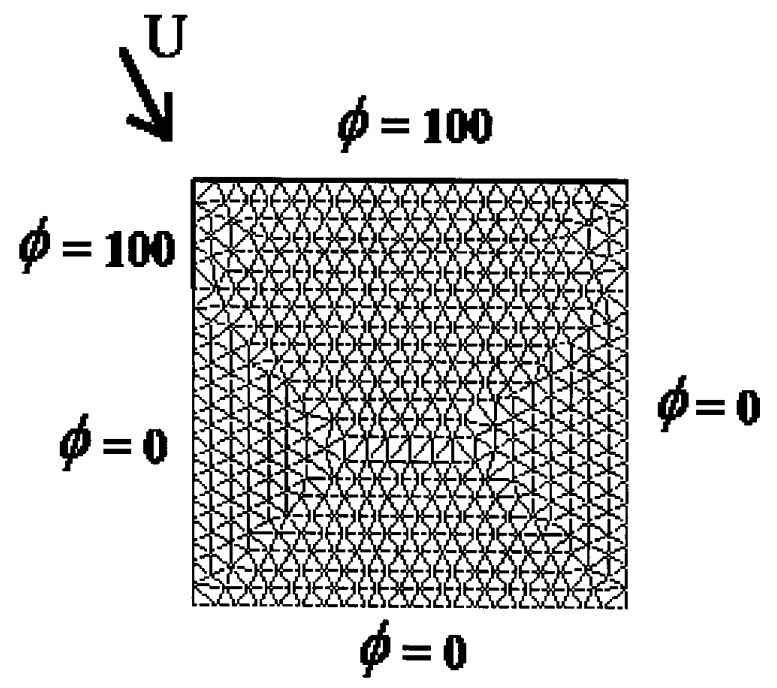

Figure 10(a). Example 4. Two-dimensional advective-diffusive problem with zero source and non-uniform Dirichlet boundary conditions. Geometry and unstructured finite element mesh of 812 linear triangles 
Distribution of $\phi$, first iteration

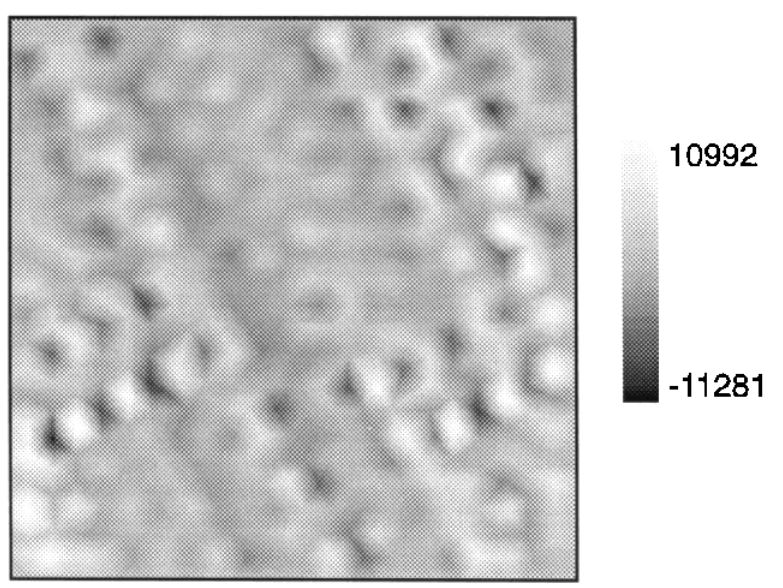

Distribution of $\phi$, fifth iteration

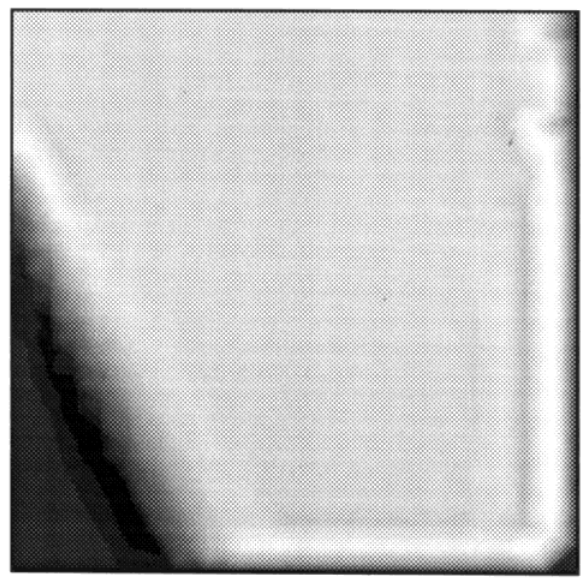

Distribution of $\phi_{1}$ tenth iteration

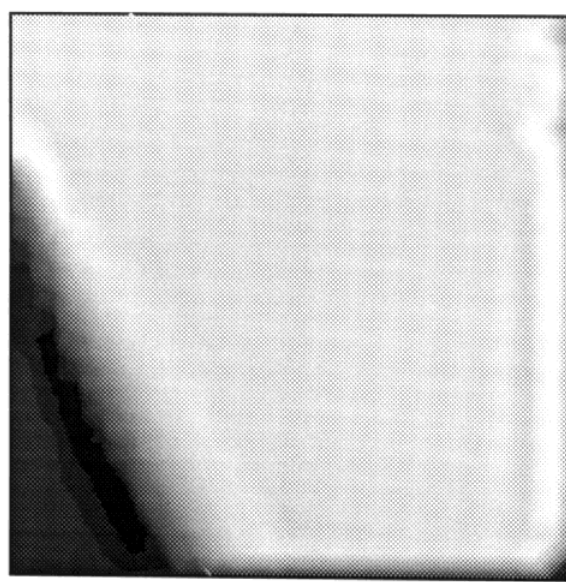

Figure 10(b). Example 4. Evolution of distribution of $\phi$ for different iterations
163.580

$-7.572$

144.910

$-6.742$ 
Distribution of $\alpha$, first iteration

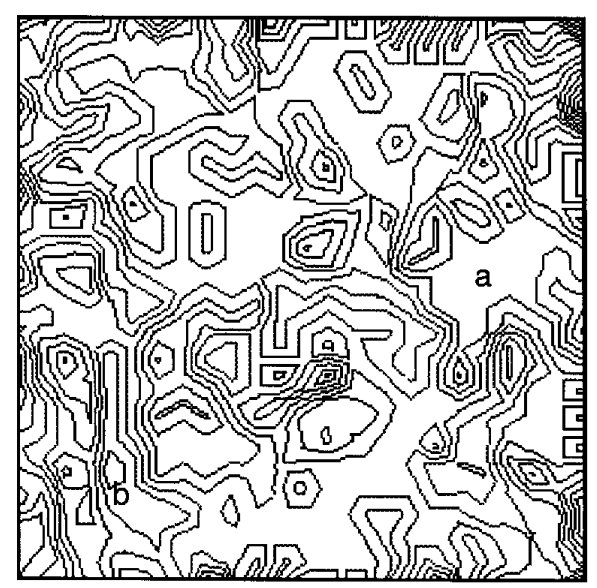

a 0.500

b 0.000

Distribution of $\alpha$, fifth iteration

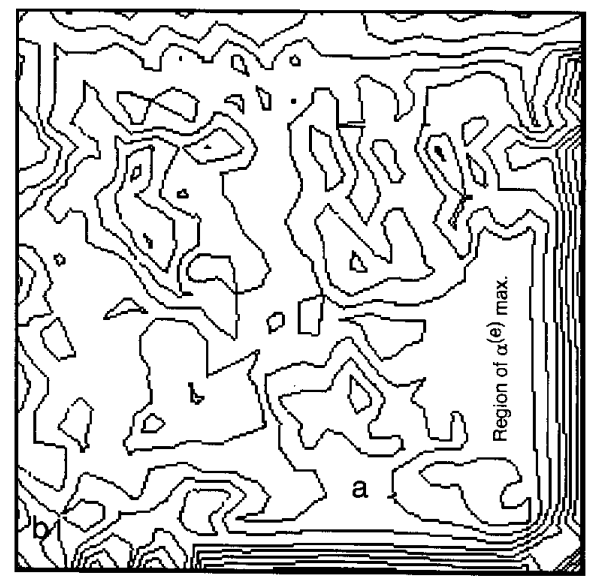

a 0.907

b 0.450

Distribution of $\alpha$, tenth iteration

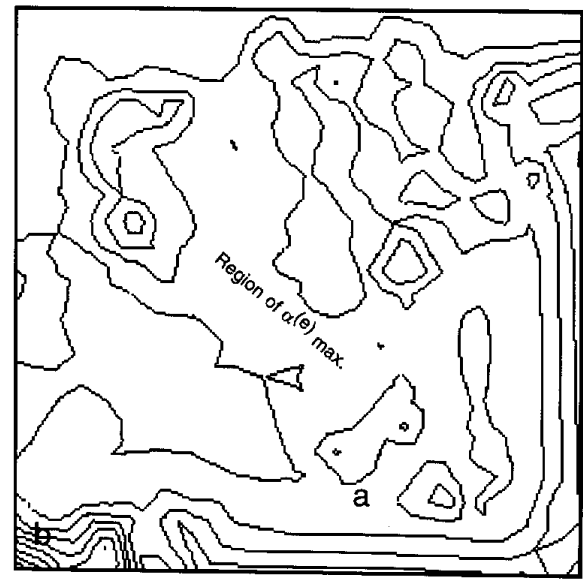

a 0.986

b 0.477

Figure 10(c). Example 4. Evolution of distribution of element stabilization parameter for different iterations 


\section{CONCLUSIONS}

The stabilized form of the governing differential equations proposed in Reference 27 provides the basic residual expression for computing iteratively the stabilization parameter for advective-diffusive problems. Extensions of the method to fluid flow problems are straightforward following the stabilized equations given in References 27 and 28. The stabilization approach proposed can be considered as a class of adaptative method where the numerical solution is sequentially enhanced by means of improving the value of the stabilization parameter while keeping the mesh and the finite element approximation unchanged. The efficiency of this ' $\alpha$-adaptativity' procedure has been shown in a number of examples of application. Further extensions of the method to a wider class of fluid flow problems are in progress.

\section{REFERENCES}

1. C. Hirsch, Numerical Computations of Internal and External Flow, Vol. 2, Wiley, New York, 1990.

2. O. C. Zienkiewicz and R. L. Taylor, The Finite Element Method, McGraw-Hill, New York, Vol. I, 1989, Vol. II, 1991.

3. D. W. Kelly, S. Nakazawa, O. C. Zienkiewicz and J. C. Heinrich, 'A note on upwind and anisotropic balancing dissipation in finite element approximation to convective diffusion problems', Int. j. numer. methods eng., 15, 1705-1711 (1980).

4. J. C. Heinrich, P. S. Hayakorn and O. C. Zienkiewicz, 'An upwind finite element scheme for two dimensional convective transport equations', Int. j. numer. methods eng., 11, 131-143 (1977).

5. A. Brooks and T. J. R. Hughes, 'Streamline upwind/Petrov-Galerkin formulation for convection dominated flows with particular emphasis on the incompressible Navier-Stokes equations', Comput. Methods Appl. Mech. Eng., 32, 199-259 (1982).

6. T. J. R. Hughes and M. Mallet, 'A new finite element formulation for computational fluid dynamics: III. The generalized streamline operator for multidimensional advective-diffusive systems', Comput. Methods Appl. Mech. Eng., 58, 305-328 (1986).

7. R. Codina, 'A finite element model for incompressible flow problems', Ph.D. Thesis, Universidad Politécnica de Catalunya, Barcelona, 1992.

8. L. P. Franca, S. L. Frey and T. J. R. Hughes, 'Stabilized finite element methods: I. Application to the advective-diffusive model', Comput. Methods Appl. Mech. Eng., 95, 253-276 (1992).

9. L. P. Franca and S. L. Frey, 'Stabilized finite element methods: II. The incompressible Navier-Stokes equations', Comput. Methods Appl. Mech. Eng., 99, 209-233 (1992).

10. I. Harari and T. J. R. Hughes, 'Stabilized finite element methods for steady advection-diffusion with production', Comput. Methods Appl. Mech. Eng., 115, 165-191 (1994).

11. T. J. R. Hughes, L. P. Franca and G. M. Hulbert, 'A new finite element formulation for computational fluid dynamics: VIII. The Galerkin/least-squares method for advective-diffusive equations', Comput. Methods Appl. Mech. Eng., 73, 173-189 (1989).

12. M. Storti, N. Nigro and S. Idelsohn, 'Equal-order interpolations: a unified approach to stabilise the incompressible and advective effects', Comput. Methods Appl. Mech. Eng., 143, 317-331 (1997).

13. S. Idelsohn, M. Storti and N. Nigro, 'Stability analysis of mixed finite element formulation with special mention to stabilized equal-order interpolations', Int. j. number. methods eng., 20, 1003-1022 (1995).

14. J. Donea, 'A Taylor-Galerkin method for convective transport problems', Int. j. numer. methods eng., 20, 101-119 (1984).

15. O. C. Zienkiewicz and R. Codina, 'A general algorithm for compressible and incompressible flow. Part I: The split characteristic based scheme', Int. j. numer. methods fluids, 20, 869-885 (1995).

16. O. C. Zienkiewicz, K. Morgan, B. V. K. Satya Sai, R. Codina and M. Vázquez, 'A general algorithm for compressible and incompressible flow. Part II: Tests on the explicit form', Int. j. numer. methods fluids, 20, 886-913 (1995).

17. R. Codina, 'Comparison of some finite element methods for solving the diffusion-convection-reaction equation', Publ. CIMNE 101, Barcelona, 1996

18. J. C. Heinrich, 'On quadratic elements in finite element solutions of steady state convection-diffusion equations', Int. $j$. numer. methods eng., 15, 1041-1052 (1980).

19. R. Codina, E. Oñate and M. Cervera, 'The intrinsic time for the streamline upwind Petrov-Galerkin formulation using quadratic elements', Comput. Methods Appl. Mech. Eng., 94, 239-262 (1992).

20. S. Idelsohn, N. Nigro, M. Storti and G. Buscaglia, 'A Petrov-Galerkin formulation for advection-reaction-diffusion', Comput. Methods Appl. Mech. Eng., 36, 27-46 (1996).

21. S. Idelsohn, 'Upwind techniques via variational principles', Int. j. numer. methods eng., 28, 669-784 (1989).

22. T. J. R. Hughes, 'Multiscale phenonema: Green's functions, subgrid scale models, bubbles and the origins of stabilised methods', Comput. Methods Appl. Mech. Eng., 127, 387-401 (1995).

23. F. Brezzi, M. O. Bristeau, L. P. Franca, M. Mallet and G. Rogé, 'A relationship between stabilized finite element methods and the Galerkin method with bubble functions', Comput. Methods Appl. Mech. Eng., 96, 117-129 (1992). 
24. F. Brezzi and A. Russo, 'Choosing bubbles for advection-difusion problems', Math. Models Meth. Appl. Sci. 4, 571-587, 1994.

25. F. Brezzi, D. Marini and A. Russo, 'Pseudo residual-free bubbles and stabilized methods', in J. A. Desiden, C. Hirscle, P. Le Tallec, E. Oñate, M. Pandolfi, J. Periaux and E. Stein (eds), Computational Methods in Applied Sciences '96, pp. 3-9, Wiley, New York, 1996.

26. F. Brezzi, L. P. Franca, T. J. R. Hughes and A. Russo, ' $b=\int g$ ', UCD/CCM Rep. 81, University of Colorado, Denver, CO, 1996. To be published in Comput. Meth. Appl. Mech. Engng

27. E. Oñate, 'Derivation of stabilized equations for advective-diffusive transport and fluid flow problems', Comput. Methods Appl. Mech. Eng., in press.

28. E. Oñate, 'On the stabilization of numerical solutions for advective-diffusive transport and fluid flow problems', Publ. CIMNE 81, Barcelona, 1996.

29. R. Codina, 'A discontinuity-capturing crosswing-dissipation for the finite element solution of the convection-diffusion equations', Comput. Methods Appl. Mech. Eng., 110, 325-342 (1993).

30. O. C. Zienkiewicz and J. Z. Zhu, 'The superconvergent patch recovery (SPR) and adaptive finite element refinement', Comput. Methods Appl. Mech. Eng., 101, 207-224 (1992). 